\title{
Clinical Outcomes of 103 Hand-Assisted Laparoscopic Surgeries for Left-Sided Colon and Rectal Cancer: Single Institutional Review
}

\author{
Narimantas Evaldas Samalavicius, Rakesh Kumar Gupta ${ }^{1}$, Audrius Dulskas ${ }^{2}$, Darius Kazanavicius ${ }^{2}$, \\ Kestutis Petrulis ${ }^{2}$, Raimundas Lunevicius ${ }^{3}$ \\ Center of Oncosurgery, Institute of Oncology, Vilnius University, Clinic of Internal, Family Medicine and Oncology, Faculty of Medicine, \\ Vilnius, Lithuania; ${ }^{1}$ Department of Surgery, Gastrointestinal Unit, B.P. Koirala Institute of Health Sciences, Dharan, Nepal; ${ }^{2}$ Vilnius University, \\ Institute of Oncology, Vilnius, Lithuania; ${ }^{3}$ Aintree University Hospitals NHS Foundation Trust, University Hospital Aintree, Liverpool, UK
}

Purpose: The laparoscopic colectomy is avoided principally because of its technical difficulty, steep learning curve, and increased operative time. Hand-assisted laparoscopic surgery (HALS) is an alternative technique that addresses these problems while preserving the short-term benefits of a laparoscopic colectomy. Our study was aimed to describe the characteristics of patients admitted due to left-sided colon and rectal cancer for HALS.

Methods: A prospectively maintained database was used to identify patients who underwent HALS at the Institute of Oncology, Vilnius University, from July 1, 2009, to October 1, 2012.

Results: One hundred-three HALS colorectal resections were performed. The patients' mean age was $64 \pm 13.4$ years. There were 46 male and 57 female patients. The body mass index was $27.3 \pm 5.8 \mathrm{~kg} / \mathrm{m}^{2}$. Forty-three patients $(41.8 \%)$ had experienced prior abdominal surgery. The mean HALS time was 105 minutes (range, 55-185 minutes). The conversion rate was $2.7 \%$ (3/103). The median of return of gastrointestinal function was 2.5 days (range, 2.2-4.5 days). The median length of hospital stay was 9 days. The postoperative complication and mortality rates were $10.7 \%$ and $0.97 \%$, respectively. Four incisional hernias $(3.9 \%)$ were seen at a mean follow-up of $7.0 \pm 3.4$ months. None of the patients had a trocar or a hand-port site recurrence.

Conclusion: A HALS colorectal resection is a safe and effective technique, and it provides all the benefits of minimally invasive surgery.

Keywords: Hand-assisted laparoscopy; Laparoscopic colectomy; Short-term outcomes

Received: August 14,2013 • Accepted: September 23, 2013

Correspondence to: Rakesh Kumar Gupta, M.S.

Department of Surgery, Gastrointestinal Unit, B.P. Koirala Institute of Health

Sciences, Dharan-18, 56700 Nepal

Tel: +977-9842040265, Fax: +977-25-520251

E-mail: rakesh154@yahoo.co.in

This original article was presented at International Society of University Colon and Rectal Surgeons Interim Meeting in Vilnius 2013-World Colorectal Conference, 31st May-1st June, 2013, Vilnius, Lithuania.

(C) 2013 The Korean Society of Coloproctology

This is an open-access article distributed under the terms of the Creative Commons Attribution NonCommercial License (http://creativecommons.org/licenses/by-nc/3.0) which permits unrestricted noncommercial use, distribution, and reproduction in any medium, provided the original work is properly cited.

\section{INTRODUCTION}

A laparoscopic colectomy (LAC) was first reported by Jacobs et al. [1] in 1991. Numerous comparative studies of a LAC vs an open colectomy for both benign and malignant conditions have demonstrated many short-term clinical benefits of the LAC, including less postoperative pain, fewer wound and pulmonary complications, decreased need for blood transfusion, faster return of bowel function, and decreased length of hospital stay $[2,3]$. It is noteworthy that randomized controlled trials have shown equivalent oncologic outcomes [4].

Despite having all the benefits of laparoscopic surgery, adoption of the LAC has been relatively slow. The LAC was estimated to ac- 
count for only less than $10 \%$ of colectomies [5]. The adoption of the laparoscopic approach has not been as rapid for the colectomy as it was for the cholecystectomy because laparoscopic colon surgery is associated with a steep learning curve due to the need to work in all four abdominal quadrants on a mobile target and to expose (and ligate) substantial vascular structures and due to the possible challenge of forming an intracorporeal anastomosis [6]. The estimated learning curve is between 25 and 60 cases, depending on the level of complexity [7]. For the majority of general surgeons, that number of colectomies would equal or exceed their annual colectomy volume.

Hand-assisted laparoscopic surgery (HALS) is a technique that was developed soon after the introduction of general laparoscopic surgery-that is, in the mid-1990s [8]. Although this technique was met by fierce resistance from the laparoscopic community, it is now gaining popularity as an adjunct and a bridge towards total laparoscopic colorectal surgery [9]. It seemingly bridges the gap between open surgery and a LAC, and that might widen the appeal of the benefits of laparoscopic surgery by rendering the procedure easier to perform. This is because a porthole-like device is inserted in the abdominal wall, which allows the surgeon's hand to be placed into the abdominal cavity while preserving pneumoperitoneum. The surgeon's hand, therefore, can work in concert with standard laparoscopic cameras and instrumentation to palpate intraabdominal structures and to assist in dissection, retraction, and control of bleeding. This means that the surgeon's hand, placed intra-abdominally, facilitates the operation, thereby increasing the ease and speed. The 6 - to $7-\mathrm{cm}$-long hand port serves as the extraction site for the specimen.

The study aims to describe the characteristics of patients admitted due to left-sided colon and rectal cancer for HALS in a single institution, the colorectal resections performed, the perioperative variables, and the short-term and long-term clinical outcomes. The prospects for HALS are discussed.

\section{METHODS}

This study was a retrospective analysis of prospectively-collected data in a single tertiary care institution. A prospectively-maintained database was used to identify all patients who underwent HALS for left-sided colon and rectal cancer at the Institute of Oncology, Vilnius University, from July 1, 2009, when HALS using transumbilical hand port incision was started, to October 1, 2012. All consenting patients aged 18 years or older with histologicallyconfirmed invasive cancers of the descending colon, the sigmoid colon, as well as the upper and the middle rectum, were included in this study. There was a single exclusion criterion-a carcinoma in situ. The following variables were included in the final HALS database: age, sex, body mass index, comorbidities, cancer location and stage, prior abdominal surgery, the operation performed, operative time, estimated blood loss, intraoperative complication, conversion, time to return of gastrointestinal function, length of hospital stay, postoperative complication within 30 days, and up to 30 months HALS incision and trocar site follow-up outcomes.

Conversion to an open procedure was defined as lengthening of the hand-port incision more than what was originally planned in order to perform the procedure. Length of hospital stay was defined as the number of nights the patient spent from the day of surgery. Return of gastrointestinal function was defined as the postoperative day when the patient tolerated a soft diet and passed stool.

\section{Surgical technique}

HALS was performed in a standardized manner. Under general anesthesia with the patient in a supine horizontal position with legs outstretched, the body fixed to the operating table and the surgeon standing between the outstretched legs, a 6- to $6.5-\mathrm{cm}$ long transumbilical incision was performed for the Dextrus Endopath (Ethicon Endo-Surgery LLC, Guaynabo, Puerto Rico) hand-port device insertion. The HALS resection was accomplished with this hand port and three additional ports. The locations of the three trocars were standard - 10- and 12-mm trocars on the right side and one 5-mm trocar on the left side (Fig. 1).

Mobilization begins with the descending colon being moved upwards to the splenic flexure and the left side of transverse colon by using the hand and a harmonic scalpel. After this, the mobilization continues with the sigmoid colon; then, the rectosigmoid is lifted at the level of the promontorium with superior rectal vessels. Continuous visualization of the left ureter is the critical part of the dissection. Then, the inferior mesenteric artery is mobilized and ligated using titanium 10-mm clips 1 to $2 \mathrm{~cm}$ from the aorta, and mobilization of the inferior mesenteric vein and its ligation continue at the level of the ligament of Treitz. The specimen is di-

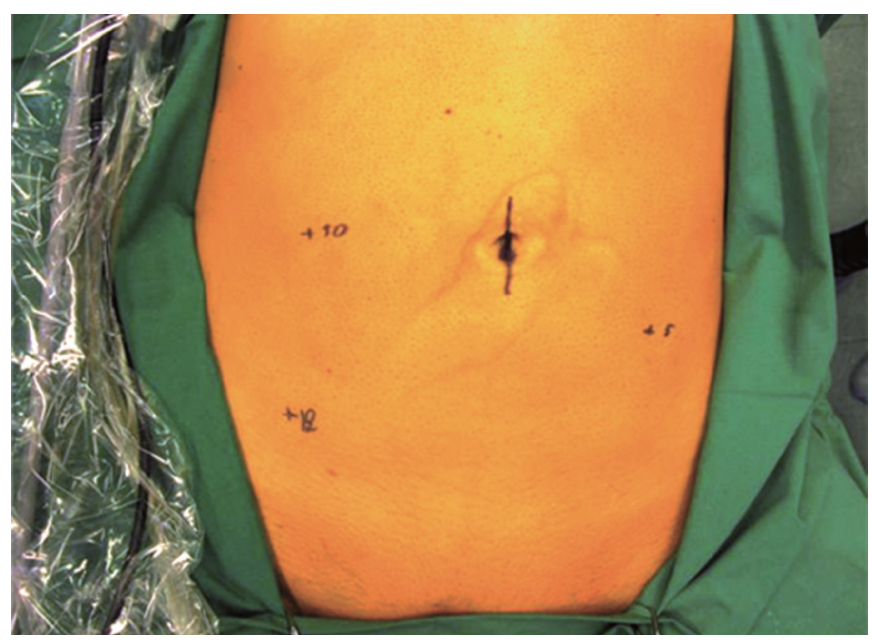

Fig. 1. A 6- to 6.5-cm-long transumbilical incision for Dextrus Endopath (Ethicon Endo-Surgery LLC, Guaynabo, Puerto Rico) handport device insertion and standard locations of the three trocars: the 10 - and the $12-\mathrm{mm}$ trocars on the right and the $5-\mathrm{mm}$ trocar on the left side of the abdominal wall. 
vided using an endoscopic linear stapler at the level of the promontorium for the left hemicolectomy or sigmoidectomy and is divided $5 \mathrm{~cm}$ below the lower edge of the tumor in the mesorectal excision for upper or middle rectal cancer. The specimen is removed through the hand-port incision, and a further anastomosis is performed laparoscopically using a double stapling technique, with a water-air leak test being performed and the rings from the stapler being examined for integrity. A drain is routinely placed only after an anterior rectal resection with a mesorectal excision and is removed on postoperative day 2 to 5 . The fascia is closed at the level of the 12-mm trocar with a single interrupted suture and at the level of the hand port with a running Polydioxanone (PDS) 0 suture. Skin incisions are closed with interrupted sutures.

\section{Statistics}

Data were entered, calculated and analyzed in Microsoft Office Excel 2007. We report most analyses as simple descriptive statistics with a standard deviation unless otherwise specified. The operative time's trend was explored from a scatter chart. This project was approved by the Vilnius Oncology Institute Ethical Review Board.

Table 1. HALS procedures performed in 103 patients

\begin{tabular}{lc}
\hline HALS procedure & No. (\%) \\
\hline Anterior rectal resection with partial mesorectal excision & $42(40.8)$ \\
Left hemicolectomy & $40(38.8)$ \\
Sigmoid colectomy & $15(14.5)$ \\
Anterior rectal resections with total mesorectal excision & $4(3.9)$ \\
Subtotal colectomy & $2(1.9)$ \\
\hline
\end{tabular}

Table 2. Intraoperative outcomes and cancer staging: 103 hand-assisted laparoscopic surgeries for left-sided colon and rectal cancer

\begin{tabular}{lc}
\hline Variable & Value \\
\hline Operative time (min) & $105(55-185)$ \\
Estimated blood loss (mL) & $120(60-270)$ \\
Intraoperative complication $^{\mathrm{a}}$ & $2(1.9)$ \\
Conversion & $3(2.7)$ \\
\multicolumn{1}{c}{ Due to massive adhesions } & $1(0.97)$ \\
Due to penetrating T4 cancer & $1(0.97)$ \\
Due to unexpected cancer location ${ }^{\mathrm{b}}$ & $1(0.97)$ \\
Length of specimen (cm) $^{\text {No. of lymph nodes harvested }}$ & $19.5(8-95)$ \\
\hline
\end{tabular}

Values are presented as mean (range) or number (\%).

${ }^{a} \mathrm{~A}$ sigmorectal anastomotic defect in a staple line following anterior rectal resection was observed and proven by using an air-leak test; the defect was closed by using an interrupted single layer of sutures. ${ }^{\text {TT}}$ The preoperative diagnosis was descending colon cancer; however, cancer was found in the splenic flexure.

\section{RESULTS}

\section{Characteristics}

Over a 36-month period, 103 HALS colorectal resections were performed. Overall, the patients' average age was $64 \pm 13.4$ years (range, 32-89 years). There were 46 male and 57 female patients. The mean body mass index was $27.3 \pm 5.8 \mathrm{~kg} / \mathrm{m}^{2}$ (range, $22-36$ $\left.\mathrm{kg} / \mathrm{m}^{2}\right)$. Seventy patients $(68.0 \%)$ had comorbidities: $65(63.1 \%)$ cardiac, 9 pulmonary, 9 diabetes, and 4 renal. Six patients had other various comorbidities; however, the majority of patients (72.8\%) were designated as American Society of Anesthesiologists physical status 1 or 2. Forty-three patients (41.8\%) had experienced a prior abdominal surgery.

\section{Diagnosis}

All patients had invasive left-sided colon or rectal cancer. Diagnoses included, in descending order, sigmoid colon cancer for 49 patients (47.6\%), upper rectal cancer for 42 patients (40.8\%), descending colon cancer for seven patients $(6.8 \%)$, middle rectal cancer for four patients (3.9\%), and colon splenic flexure cancer for one patient $(0.9 \%)$. Stage I cancer was confirmed for 27 patients (26.2\%), stage II for 29 (28.1\%), stage III for 39 (37.9\%), and stage IV for $9(8.7 \%)$.

\section{HALS procedures}

The procedures performed are shown in Table 1. Anterior rectal resections with partial mesorectal excision were performed when cancers in the rectum were above $12 \mathrm{~cm}$ from the dentate line. Low anterior rectal resections with total mesorectal excision were performed for middle rectal cancer. One subtotal colectomy with ileorectal anastomosis was performed due to sigmoid cancer and familial adenomatous polyposis, and another subtotal colectomy was performed due to descending colon cancer and multiple polyps in the transverse and right colon. Two patients underwent a HALS sigmoid colectomy, one at 9 and the other at 10 days, after a laparoscopic sigmoid colotomy and polypectomy for large sigmoid adenomas with high-grade dysplasia, which in surgical specimen histology turned out to be T1 sigmoid colon cancer;

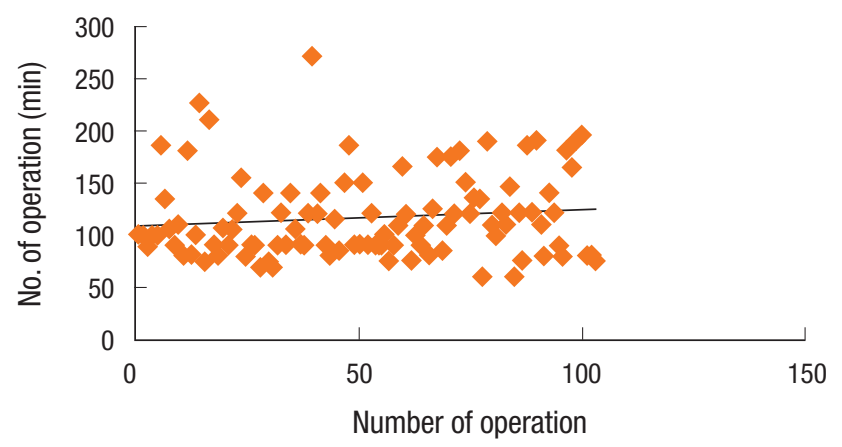

Fig. 2. Trend of operative time educed from the first 103 hand-assisted laparoscopic surgeries for left-sided colon and rectal cancer. 
Table 3. Primary HALS and postoperative complications

\begin{tabular}{lcccc}
\hline Primary operation & Complication & Patient, $\mathrm{n}(\%)$ & Management & Outcome \\
\hline ARR with PME & Anastomotic leak & $1(0.9)$ & Laparotomy, washout, loop ileostomy & Recovered \\
Left hemicolectomy & Paracolic abscess ${ }^{\mathrm{a}}$ & $1(0.9)$ & Laparotomy, washout, loop ileostomy & Recovered \\
ARR with PME & Urinary retention & $2(1.9)$ & Suprapubic catheter & Recovered \\
ARR with PME & Bleeding from the anastomotic line & $1(0.9)$ & Conservative & Recovered \\
Subtotal colectomy & Stroke & $1(0.9)$ & Conservative & Recovered \\
ARR with PME & Myocardial infarction & $1(0.9)$ & Conservative & Recovered \\
Left hemicolectomy & Subacute intestinal obstruction & $3(2.7)$ & Conservative & Recovered \\
ARR with PME: conversion to open & Septic pneumonia & $1(0.9)$ & Conservative & Died \\
\hline
\end{tabular}

HALS, hand-assisted laparoscopic surgery; ARR, anterior rectal resection; PME, partial mesorectal excision.

aDue to perforation above the anastomotic line.

none had residual or lymph-node disease in final pathology.

\section{Intraoperative outcomes}

Intraoperative outcomes are shown in Table 2. It is noteworthy to mention that the mean HALS time is 105 minutes. The operating time increased only slightly within the defined period of 2010-2012 (Fig. 2). However, there was no statistical significant difference when the operating time was compared within groups of patient operated on in the years of 2010,2011, and 2012. There were no episodes of significant intraoperative bleeding. There was a positive air-leak test in two patients (1.9\%), and interrupted 3.0 vicryl sutures were additionally used to secure the anastomosis. The conversion rate was $2.7 \%(3 / 103)$. The reason for conversion was massive adhesions $(0.97 \%)$, penetrating T4 cancer $(0.97 \%)$, and unexpected cancer $(0.97 \%)$. The average number of lymph nodes harvested was 16, with the maximum number being 40 .

\section{Postoperative period outcomes}

The median of return of gastrointestinal function was 2.5 days (range, 2.2-4.5 days). The median length of hospital stay was 9 days (range, 3-31 days). The postoperative complication rate was 10.7\% (11 patients). Postoperative complications following HALS and their consequences are shown in Table 3. Two patients (1.9\%) required an explorative laparotomy. The postoperative mortality rate was $0.97 \%$ (one death). A 7-year-old male patient who underwent a partial mesorectal excision for stage III upper rectal cancer died because of septic pneumonia on the 7th postoperative day.

\section{HALS incision and trocar sites follow-up}

There were 4 incisional hernias (3.9\%) seen on a mean followup of 7.0 \pm 3.4 months (range, 2-30 months). None of the patients had trocar or hand-port site recurrence.

\section{DISCUSSION}

The indications of HALS have been extended successfully for a broad range of disease. Although used for all types of colectomies, segmental colectomies represented the most common procedure, with a significant percentage being left-sided or rectal resections [10]. As our institution is a tertiary oncological center, most of our HALS patients underwent surgery due to cancer. Patients with descending colon, sigmoid, and upper rectal cancer, are ideal candidates for this technique. This is attributed to the fact that leftsided colonic cancers are more common than right-sided ones in our catchment area. Furthermore, most right-sided tumors are dealt with by general surgeons in nearby secondary general hospitals while patients with left-sided, and especially rectal, cancer are usually referred to our institution for management. Although in the literature, HALS is used for a right hemicolectomy, we do not use this approach for a right hemicolectomy, as we do not see much advantage there because the anastomosis is done extracorporeally after a right hemicolectomy.

With increasing experience, we performed HALS for more complex colon procedures, including subtotal colectomies with ileorectal anastomosis. It is important to emphasize that the mean operative time was only 105 minutes in our series (range, 55185). Also, the trend of the operative time was almost horizontal, suggesting that HALS colorectal resections for left-sided large bowel cancer is not a big technical challenge for surgeons who are quite familiar with general colorectal surgery techniques and have had general laparoscopic training [11]. On the other hand, we feel that HALS operating times decreased in the year 2012 without any negative consequences. Also, this decrease was similar to those of others who have reported decreased operative times for HALS $[11,12]$ compared with LAC, while maintaining much of the short-term outcome benefits and morbidity as compared with LAC [13-15]. We should note that, as only 4 patients with middle rectal cancer were included in these series, our experience allows us to emphasize that this surgical technique is indicated for leftsided colonic and upper rectal cancer.

There have been a number of small reports on a HALS colectomy and 2 randomized controlled trials of a HALS colectomy compared with LC that only evaluated 74 HALS cases [16, 17]. When the short-term patient outcomes of a HALS colectomy and 
a LAC are compared, they are found to be similar $[2,16]$. However, the conversion rate is a less suitable variable for a HALS analysis, as conversion is required infrequently. The conversion rate in our study $(2.7 \%)$ is less than the $3-12 \%$ conversion rate reported in other studies $[16,17]$.

We also found in this study that our postoperative complication rate was similar to those published in the HALS studies and was comparable to the reported LAC experience [16-18]. However, there were significant differences between the HALS and the LAC, including decreased operative times and fewer converted procedures in the HALS cases. In a multicenter, prospective, randomized trial comparing HALS and LAC for left-sided segmental and total colectomies, there were significant reductions in operative times for both segmental and total colectomies in favor of HALS [19].

In our series, the HALS device was inserted in the midline because the mobilized colon is a midline structure. It also keeps the lateral abdomen free of incisions should an ostomy ever become an issue, and it allows for easy conversion to an open procedure if necessary. In fact, most of the conversions in this study only required a small extension of the HALS incision.

The cost of a new technology needs to be considered in the current healthcare system. The economic considerations of LAC and HALS colectomies have been analyzed in various studies [20, 21]. A comparative study of 100 HALS colectomies to LAC demonstrated that, although the costs of operating-room supplies were higher in the HALS cases, there was no difference in the hospitalization costs [20]. In most institutions where operating-room costs are allocated in fractions of an hour, a 30- to 60-minute decrease in operating time could represent a significant financial savings for the institution.

There were no trocar site or HAL incision site recurrence in any of our patients. Although trocar site recurrence would be a concern [22], a recent prospective study comparing a laparoscopic with an open colectomy for cancer did not show any difference in survival between the two groups [23], and a randomized multicenter trial demonstrated oncological noninferiority for the laparoscopic approach [4]. Most HAL devices function as wound protectors, which should theoretically protect the HAL wound from tumor implantation.

The long-term complications of HALS have been the center of recent debate. It has been postulated that a continuous and persistent stretch of the port site may lead to the development of an incisional hernia. Furthermore, placement of the hand in the abdomen in HALS increases the risk of postoperative ileus and the development of intra-abdominal adhesions with a future risk of small bowel obstruction [24]. In our series, with a follow-up of up to 30 months, an incisional hernia was confirmed to $3.9 \%$ of patients. Three patients had a subacute intestinal obstruction following a left hemicolectomy (2.7\%) within 30 postoperative days. However, no patients had a small bowel obstruction afterwards.

In summary, this study of a diverse colorectal practice for more than three years provides insight into the applicability and the outcome of HALS compared to colorectal resections. The HALS approach to left-sided colonic and upper rectal cancer is safe and effective, and has outcomes similar to those published for laparoscopic colorectal surgery. In the present series, no obvious drawbacks for HALS colorectal surgery have been identified. As a quality-related outcome, there was no learning curve for this study. Rather, acceptable HALS outcomes were achieved from the outset. Thus, concerns about initial quality-related outcomes should not be an obstacle to surgeons who are considering the adoption of this technique. Increased use of HALS could increase the number of patients who would benefit from minimal-access colon and rectal resections.

In conclusion, the HALS technique provides all the benefit of minimally invasive surgery for patients who undergo colorectal resections due to left-sided colon and upper rectal cancer. The HALS colorectal resection is a safe and effective procedure.

\section{CONFLICT OF INTEREST}

No potential conflict of interest relevant to this article was reported.

\section{REFERENCES}

1. Jacobs M, Verdeja JC, Goldstein HS. Minimally invasive colon resection (laparoscopic colectomy). Surg Laparosc Endosc 1991;1: 144-50.

2. Noel JK, Fahrbach K, Estok R, Cella C, Frame D, Linz H, et al. Minimally invasive colorectal resection outcomes: short-term comparison with open procedures. J Am Coll Surg 2007;204:291307.

3. Janson M, Bjorholt I, Carlsson P, Haglind E, Henriksson M, Lindholm E, et al. Randomized clinical trial of the costs of open and laparoscopic surgery for colonic cancer. Br J Surg 2004;91:409-17.

4. Clinical Outcomes of Surgical Therapy Study Group. A comparison of laparoscopically assisted and open colectomy for colon cancer. N Engl J Med 2004;350:2050-9.

5. Steele SR, Brown TA, Rush RM, Martin MJ. Laparoscopic vs open colectomy for colon cancer: results from a large nationwide population-based analysis. J Gastrointest Surg 2008;12:583-91.

6. Schirmer BD. Laparoscopic colon resection. Surg Clin North Am 1996;76:571-83.

7. Tekkis PP, Senagore AJ, Delaney CP, Fazio VW. Evaluation of the learning curve in laparoscopic colorectal surgery: comparison of right-sided and left-sided resections. Ann Surg 2005;242:83-91.

8. Ou H. Laparoscopic-assisted mini laparatomy with colectomy. Dis Colon Rectum 1995;38:324-6.

9. Ballantyne GH, Leahy PF. Hand-assisted laparoscopic colectomy: evolution to a clinically useful technique. Dis Colon Rectum 2004; 47:753-65.

10. Cima RR, Pattana-arun J, Larson DW, Dozois EJ, Wolff BG, Pemberton JH. Experience with 969 minimal access colectomies: the 
role of hand-assisted laparoscopy in expanding minimally invasive surgery for complex colectomies. J Am Coll Surg 2008;206: 946-50.

11. Pendlimari R, Holubar SD, Dozois EJ, Larson DW, Pemberton $\mathrm{JH}$, Cima RR. Technical proficiency in hand-assisted laparoscopic colon and rectal surgery: determining how many cases are required to achieve mastery. Arch Surg 2012;147:317-22.

12. Ozturk E, da Luz Moreira A, Vogel JD. Hand-assisted laparoscopic colectomy: the learning curve is for operative speed, not for quality. Colorectal Dis 2010;12(10 Online):e304-9.

13. Targarona EM, Gracia E, Garriga J, Martinez-Bru C, Cortes M, Boluda R, et al. Prospective randomized trial comparing conventional laparoscopic colectomy with hand-assisted laparoscopic colectomy: applicability, immediate clinical outcome, inflammatory response, and cost. Surg Endosc 2002;16:234-9.

14. Hand-assisted laparoscopic surgery vs standard laparoscopic surgery for colorectal disease: a prospective randomized trial. HALS Study Group. Surg Endosc 2000;14:896-901.

15. Maartense S, Bemelman WA, Gerritsen van der Hoop A, Meijer DW, Gouma DJ. Hand-assisted laparoscopic surgery (HALS): a report of 150 procedures. Surg Endosc 2004;18:397-401.

16. Aalbers AG, Biere SS, van Berge Henegouwen MI, Bemelman WA. Hand-assisted or laparoscopic-assisted approach in colorectal surgery: a systematic review and meta-analysis. Surg Endosc 2008; 22:1769-80.
17. Iqbal M, Bhalerao S. Current status of hand-assisted laparoscopic colorectal surgery: a review. J Laparoendosc Adv Surg Tech A 2007;17:172-9.

18. Schadde E, Smith D, Alkoraishi AS, Begos DG. Hand-assisted laparoscopic colorectal surgery (HALS) at a community hospital: a prospective analysis of 104 consecutive cases. Surg Endosc 2006; 20:1077-82.

19. Marcello PW, Fleshman JW, Milsom JW, Read TE, Arnell TD, Birnbaum EH, et al. Hand-assisted laparoscopic vs. laparoscopic colorectal surgery: a multicenter, prospective, randomized trial. Dis Colon Rectum 2008;51:818-26.

20. Dowson HM, Huang A, Soon Y, Gage H, Lovell DP, Rockall TA. Systematic review of the costs of laparoscopic colorectal surgery. Dis Colon Rectum 2007;50:908-19.

21. Ozturk E, Kiran RP, Geisler DP, Hull TL, Vogel JD. Hand-assisted laparoscopic colectomy: benefits of laparoscopic colectomy at no extra cost. J Am Coll Surg 2009;209:242-7.

22. Wexner SD, Cohen SM. Port site metastases after laparoscopic colorectal surgery for cure of malignancy. Br J Surg 1995;82:295-8.

23. Leung KL, Kwok SP, Lam SC, Lee JF, Yiu RY, Ng SS, et al. Laparoscopic resection of rectosigmoid carcinoma: prospective randomised trial. Lancet 2004;363:1187-92.

24. Sonoda T, Pandey S, Trencheva K, Lee S, Milsom J. Longterm complications of hand-assisted versus laparoscopic colectomy. J Am Coll Surg 2009;208:62-6. 